\title{
The Repairman Problem Revisited
}

\author{
Claude Crampes*and Jérôme Renault ${ }^{\dagger}$
}

February 16, 2015

\begin{abstract}
We use a probabilistic model to show that the development of a large number of small production units facing independent risks of failure must be accompanied by an approximately proportional increase in the number of repairmen. We derive the cost function of a machine in working condition, which increases with the cost of an installed machine and with the repair cost and failure probability, and decreases with repairman efficiency. We provide a very simple proof and generalize the results of ARRow et al. (1972). We also show that it is redundant to require installers to hire the number of repairmen necessary to reach a fixed availability level for each installed machine.
\end{abstract}

Keywords: failure, repair, Markov chain, Hicks-Leontiev technology

${ }^{*}$ Toulouse School of Economics (GREMAQ, UT1-Capitole), claude.crampes@tse-fr.eu, corresponding author

${ }^{\dagger}$ Toulouse School of Economics (GREMAQ, UT1-Capitole), jerome.renault@tse-fr.eu 


\section{Introduction}

In most industries, the scale of production at a given location has increased considerably over the past century. With larger, more powerful and more reliable machines, firms have drastically decreased their operating costs, in particular because of savings on maintenance. By contrast, some activities require local service provision on a small scale, which means a decentralized organization for maintenance. For instance, this is the case of washing machines, refrigerators and heating and hot water systems. There are also industries that switch back to small-scale activities after running huge plants for decades. This is the case of the electricity industry where environmental concern is now leading technology changes. Since the beginning of the millennium, we have been witnessing a momentum in favor of small decentralized production units, in particular those using solar and wind energies. In all the industries with small-scale production units, for a given state of the technology the only way to increase production is to increase the number of machines. Multiplying machines changes the risk of failure in a way that depends on the correlation between individual risks. It also changes the way in which repair and maintenance services are organized. Knowing the relation between the number of installed machines and the number of repairmen necessary to reach a given level of performance is essential to determine the actual cost of an operating machine. For example, in the case of renewable energy, the published cost of MW from wind turbines generally does not include the cost of repair, which can be high because of "faults like imbalance, wear, fatigue and impending cracks in rotor blades, bearings, shafts, in the gearbox, the generator and in the yaw- and the pitch angle mechanism". ${ }^{1}$

Whether installed machines and repairmen are substitutes or complements in the production process is an intriguing question that was explored by economists in the 1970s. ${ }^{2}$ LEVHARI and Sheshinski [1970] argued that, starting from a given combination of machines and repairmen, we can increase one of the inputs to compensate for a decrease of the other one without changing the average number of machines in working condition. Moreover, contrary to the intuition that a repair team should be viewed as a form of public good potentially generating economies of scale, LEVHARI and SHESHINSKI showed that the combination of inputs results in a technology with constant returns to scale: "We find a surprisingly good fit with the following two main characteristics: (i) Quasi-Concavity. Isoquants have the 'right' shape with decreasing marginal products for both factors (machines and repairmen). (ii) Almost Constant Returns to Scale. In none of the cases that we have considered has the absolute deviation from constant returns to scale been larger than 1.5 per cent. This is in contrast to the conjecture (raised by FELLER, [1957]) that such a random process yields increasing returns to scale because of the 'law of large numbers'" (op. cit.: 560). Later, ARRow et al. [1972] showed that result (i) depends on the scale of production and is false in so far as installed machines and repairmen are strict complements for large expected levels of production. As regards result (ii), their analysis confirms the absence of increasing returns to scale.

In Section 2, we devise a model to analyze the repairman problem in line with ARrow et al. [1972]. Our contribution, summarized in Theorem 2.2 is threefold: 1) we study a new criterion called "individual reliability" which takes care of the production level guaranteed per machine; 2) we provide an upper bound on the production, which is valid for any number of machines and repairmen; and

\footnotetext{
${ }^{1}$ HAMEed et al. $[2009]$ page 2.

${ }^{2}$ See Arrow et al. (1972), Levhari and Sheshinski (1970), Syrquin (1972)
} 
3) we give a new and much simpler proof of the main result of ARROW et al. concerning the shape of the production function for large numbers of machines and repairmen. In Section 3 we propose an heuristic proof of the results of Section 2 (the full proof can be found in the Appendix). In Section 4 we derive the economic consequences of the model, namely that having a large number of operational machine units requires a proportional combination of installed machines and repairmen. Hence, the average cost for an operating engine must add a function that includes the cost of repairmen and the probabilities of failure and repair to the purchase cost. We present our concluding remarks in Section 5.

\section{Problem, Model and Results}

The general problem addressed here is the following: Consider a large production factory with $m$ identical machines and $r$ identical repairmen. How should $m$ and $r$ be related to reach a given level of production? In this paper we follow the model of ARROW et al. [1972] but we propose a much simpler proof. Whereas their method consisted mostly of algebraic computations, ours takes advantage of the monotonic properties of the production function.

The model is as follows. There are $m$ identical and independent machines and $r$ repairmen, with $r \leq m$. At any given time, every machine is either in working mode (producing) or in failure mode, and every repairman is either servicing a machine or idle. A machine which breaks down is serviced unless all the repairmen are servicing other machines, in which case the machine joins a waiting line. We also have two fixed parameters $\lambda, \mu>0$ such that during a short interval $d t$, the probability that a working machine breaks down is equivalent to $\lambda d t$, and the probability that a machine being serviced reverts to the working mode is equivalent to $\mu d t$. We also make the standard assumption that during a short interval, either nothing happens, or exactly one machine breaks down, or else exactly one machine starts working again; in other words, simultaneous changes are negligible. To simplify our computation, we consider that at a given period all machines in the waiting line have the same probability to be serviced at this period, i.e. that repairmen choose which machine to service using a uniform distribution of probability, and naturally a machine cannot be serviced by more than one repairman at a given time.

Two main values are of interest. First, the number of machines expected to be in working order in the long run is denoted by $X(m, r)$. This represents the expected production of the factory per unit of time. Second, we would like every machine $i$ in $\{1, \ldots, m\}$ to be in working mode as often as possible, and we denote by $\alpha(m, r)$ the greatest proportion of time $\alpha$ in $[0,1]$ such that with probability one, each machine will produce at least that proportion $\alpha$ of dates in the long-run.

For each machine $i$ in $\{1, \ldots m\}$ and time $t \geq 0$ we denote by $Y_{i}^{t}$ the random variable in $\{0,1\}$ such that $Y_{i}^{t}=1$ if machine $i$ is working at time $t$, and $Y_{i}^{t}=0$ if machine $i$ is in failure mode at time $t$. We start from any initial situation, which will play no role on the limit of the trajectory followed by the system. The number of machines working at time $t$ is our main state variable

$$
n(t)=\sum_{i=1}^{m} Y_{i}^{t}
$$

which takes values in $M=\{0, \ldots, m\}$. A discrete-time modelling (with a short interval so that all 
subsequent probabilities are non-negative) leads to a homogenous Markov chain on $M$, with the following transition probabilities:

- $\mathbb{P}(n(t+1)=n(t)-1 \mid n(t))=\lambda n(t)$, because when $n$ machines are working there is probability $\lambda n$ that one of them breaks down.

- If $n(t) \leq m-r$, all repairmen are in service so we have $\mathbb{P}(n(t+1)=n(t)+1 \mid n(t))=r \mu$.

- If $n(t)>m-r$, all machines in failure mode are being serviced so we get $\mathbb{P}(n(t+1)=$ $n(t)+1 \mid n(t))=(m-n(t)) \mu$.

- Finally, the transition probabilities sum to 1 , so $\mathbb{P}(n(t+1)=n(t) \mid n(t))=1-\lambda n(t)-r \mu$ if $n(t) \leq m-r$, and $\mathbb{P}(n(t+1)=n(t) \mid n(t))=1-\lambda n(t)-(m-n(t)) \mu$ if $n(t)>m-r$.

Consequently, the transition matrix of the Markov chain $(n(t))_{t \geq 0}$ on $M$ is the following (at an entry line $i$ and column $j$ we represent the probability that the tomorrow state is $j$ if today it is $i$ ):

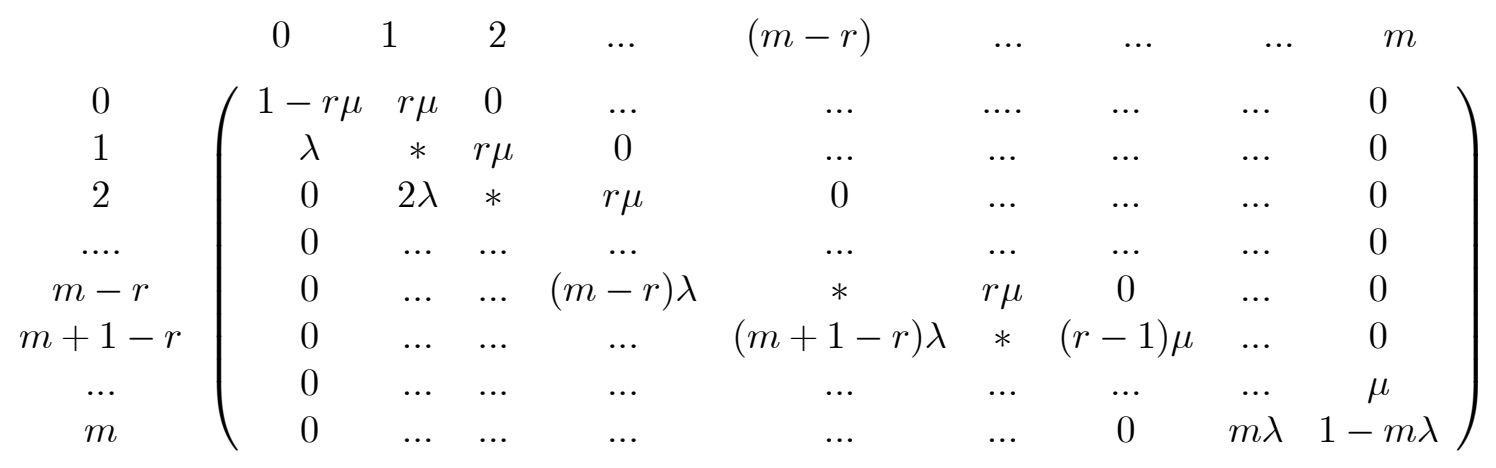

The stars denote non negative numbers such that for each row, the probabilities sum to 1 . We denote by $H$ the above stochastic matrix. It is easy to see ${ }^{3}$ that given any two states $n$ and $n^{\prime}$, and given any integer $t \geq m$, there is a positive probability of going from state $n$ to state $n^{\prime}$ in exactly $t$ stages. As a consequence, the Markov chain induced by $H$ is ergodic and there is a unique probability $p^{*}=\left(p_{0}^{*}, \ldots, p_{m}^{*}\right)$ that the matrix product $p^{*} H$ is equal to $p^{*}$. The vector $p^{*}$ is the invariant measure of the Markov chain, and for each $n$ the probability $p_{n}^{*}$ represents the time-frequency limit of exactly $n$ machines in working order: if we consider for each $t$ the iterated matrix $H^{t}$, we have geometric convergence of $H^{t}$ to the matrix $H^{*}$ with all identical rows given by $\left(p_{0}^{*}, p_{1}^{*}, \ldots, p_{m}^{*}\right)$. This means that whatever the initial state, the probability at time $t$ that exactly $n$ machines are working converges quickly to $p_{n}^{*}$ when $t$ goes to $\infty$. And the long-run expectation of the number of machines working can be expressed simply as:

$$
X(m, r)=\sum_{n=0}^{m} n p_{n}^{*}
$$

An exact computation for $X(m, r)$ can be obtained as follows. Find $p^{*}$ such that $p^{*} H=p^{*}$. This can be done by solving a linear system of $m+2$ equations with $m+1$ unknowns. Then compute $\sum_{n=0}^{m} n p_{n}^{*}$. However solving the linear system explicitly in terms of $m$ and $r$ looks difficult or maybe intractable (as pointed out by Arrow et al. p. 244, where (10) corresponds to the above equation (1) : "it seems difficult to solve (10) explicitly in terms of $m$ and $r$ "). In this paper we will provide

\footnotetext{
${ }^{3}$ This is true except for the case $r=0$. In this case, it is easy to see that eventually, all machines will break down and never be repaired, so $X(m, 0)=\alpha(m, 0)=0$. We will simply omit the case $r=0$ in the sequel.
} 
an interesting upper bound for $X(m, r)$, valid for all $m$ and $r$. We will also present an equivalent of $X(m, r)$ when the number of repairmen becomes very large, and provide a new simple proof for this result originally obtained by ARROw et al.

Finally, we take care of individual reliability. Notice that because the Markov chain is ergodic, there will be (with probability one) infinitely many periods where all machines are simultaneously broken. Consequently, we introduce and study the quantity $\alpha(m, r)$ which concerns not the number of times where machines break down, but the proportion of times when this happens. We now formally define the function $\alpha(m, r)$. For each machine $i$ and quantity $x$ in $[0,1]$, let $Z_{i}^{x}$ be the event: "it only happens finitely many times that machine $i$ has been used in the past less than a proportion $x$ of stages". Formally $Z_{i}^{x}$ can be written: $\left(\exists T_{0}, \forall T \geq T_{0}, \frac{1}{T} \sum_{t=1}^{T} Y_{i}^{t} \geq x\right)$. Now, we define:

$$
\begin{aligned}
\alpha(m, r) & =\sup \left\{\alpha \in[0,1], \forall i=1, \ldots, m, \forall x<\alpha, \mathbb{P}\left(Z_{i}^{x}\right)=1\right\}, \\
& =\sup \left\{\alpha \in[0,1], \mathbb{P}\left(\cap_{i=1, \ldots, m} \cap_{x \in[0, \alpha)} Z_{i}^{x}\right)=1\right\} .
\end{aligned}
$$

$\alpha(m, r)$ is the greatest proportion of time $\alpha$ in $[0,1]$, such that in the long run with probability one, each machine will produce at least a proportion $\alpha$ of times.

Clearly $X(m, r)$ and $\alpha(m, r)$ are non decreasing with $m$ and $r$. We are mainly interested in the case of large $r$ and $m$ and introduce the following quantity.

\section{Definition 2.1.}

$$
\varphi(m, r)=\min \left\{r \frac{\mu}{\lambda}, m \frac{\mu}{\mu+\lambda}\right\} .
$$

We will show the following results.

\section{Theorem 2.2.}

(1) $\alpha(m, r)=\frac{X(m, r)}{m}$ for all $m$ and $r$,

(2) $X(m, r) \leq \varphi(m, r)$ for all $m$ and $r$,

(3) $\lim _{r \rightarrow \infty} \frac{X(m, r)}{\varphi(m, r)}=1$.

Part (1) is indeed quite natural: imagine that the long-run expected number of machines working is the product $\alpha m$ for some $\alpha$ in $[0,1]$. Since the process is anonymous across machines, with high probability each of them will be working for approximately the same proportion of time, which cannot be anything else than $\alpha$. Formally we will prove that for any machine, the proportion of times where this machine is working converges almost surely to $\frac{X(m, r)}{m}$. The technical proof relies on probability arguments and will be examined in Subsection 6.2. Remember we have assumed that at a given period all machines in the waiting line at some period have the same probability of being serviced at this period, whereas in some cases it could be natural to assume that there is a FIFO (First In- First Out) waiting line. This would not change the result, nor would other kinds of servicing processes which are anonymous across machines, for instance a LIFO waiting line or a system where machines currently less used have service priority.

Part (2) gives an interesting upper bound on the long-run expected production. 
Part (3) can be equivalently stated as: $\forall \varepsilon>0, \exists r_{0}, \forall r \geq r_{0}, \forall m \geq r,\left|\frac{X(m, r)}{\varphi(m, r)}-1\right| \leq \varepsilon$. This may be seen as the main result, and already appears in Arrow et al., whereas Parts (1) and (2) are new. The interest of our contribution to Part (3) is that it gives a simple formal proof in Subsection 6.1, which can be described heuristically and very simply. The heuristics are presented in Section 3.

An important implication of Part (3) of the theorem is the following indication for our original question: the larger $m$ and $r$ are, the larger $X(m, r)$ and $\alpha(m, r)$ will be, but in practice there is a cost to increase $m$ or $r$ without significantly increasing $X(m, r)$ or $\alpha(m, r)$. Condition (3) tells us that as a first approximation these quantities can be replaced by $\varphi(m, r)$, and to maximize $\varphi(m, r)$ it is never necessary to have $r \frac{\mu}{\lambda}$ greater or smaller than $m \frac{\mu}{\mu+\lambda}$. So one may want to choose $m$ and $r$ such that $r \frac{\mu}{\lambda}=m \frac{\mu}{\mu+\lambda}$, that is:

$$
r=m \frac{\lambda}{\lambda+\mu}
$$

\section{A Heuristic Proof}

We proceed in four elementary steps.

Step 1. The case of $r=m$.

This is the easiest case to handle. Each machine in failure mode is being serviced, so if exactly $n$ machines are working, exactly $m-n$ machines are being serviced. For all states we have $n=0,1 \ldots, m$ :

$$
\mathbb{P}(n+1 \mid n)=(m-n) \mu \text {, and } \mathbb{P}(n-1 \mid n)=\lambda n .
$$

Notice that, starting from state $n$, the probability of going upwards (reaching state $n+1$ ) is higher than the probability of going downwards (reaching state $n-1)$ if and only if $(m-n) \mu>\lambda n$, that is $n<m \frac{\mu}{\lambda+\mu}$. So $n=m \frac{\mu}{\lambda+\mu}$ is a stationary point; at this point there is no tendency for a drift upwards or downwards, and in this case one can heuristically approximate $X(m, r)$ with $m \frac{\mu}{\lambda+\mu}$. We will prove later that this approximation is indeed fully exact here:

$$
X(m, r)=m \frac{\mu}{\lambda+\mu} \text { when } r=m .
$$

Step 2. The case of small $r$.

Either consider a small number of repairmen compared to the number of machines, that is a small $\frac{r}{m}$, or fix the value of $r$ and let $m$ go to infinity. What will the limit behavior of $X(m, r)$ be in this case ? Intuitively, all the repairmen will always be working, which is equivalent to saying that at any moment, exactly $r$ machines will be serviced.

So we make the heurisitic $\mathbb{P}(n+1 \mid n)=r \mu$ for all $n$. And as before we have $\mathbb{P}(n-1 \mid n)=$ $\lambda n$. Starting from state $n$, the probability of going upwards is higher than the probability of going downwards if and only if $r \mu>\lambda n$, that is $n<r \frac{\mu}{\lambda}$. So the stationary point is $n=r \frac{\mu}{\lambda}$, and we make the approximation:

$$
X(m, r) \approx r \frac{\mu}{\lambda} \text { when } r<<m
$$


Combining the first two cases, one can ask whether $m \frac{\mu}{\lambda+\mu}$ is greater or lower than $r \frac{\mu}{\lambda}$. Indeed, $m \frac{\mu}{\lambda+\mu}=r \frac{\mu}{\lambda}$ is equivalent to $r=m \frac{\lambda}{\lambda+\mu}$. This is an interesting intermediate case, which we will look at now.

Step 3. The case $r=m \frac{\lambda}{\lambda+\mu}$.

In this case $m-r=m \frac{\mu}{\mu+\lambda}=r \frac{\mu}{\lambda}$. For each $n$ we have $\mathbb{P}(n-1 \mid n)=\lambda n$, as before, and the probability of going upwards reads:

$$
\mathbb{P}(n+1 \mid n)=\left\{\begin{array}{cl}
m \mu \frac{\lambda}{\lambda+\mu} & \text { if } n \leq m-r \\
(m-n) \mu & \text { if } n \geq m-r
\end{array}\right.
$$

For $n \leq m-r$, we have $m \mu \frac{\lambda}{\lambda+\mu} \geq \lambda n$ so the drift is upwards, and for $n \geq m-r$ we have $(m-n) \mu \leq \lambda n$ and the drift is downwards. Consequently, the stationary point is $n=m-r$, and we make the approximation:

$$
X(m, r) \approx r \frac{\mu}{\lambda}=m \frac{\mu}{\mu+\lambda} \text { when } r=m \frac{\lambda}{\lambda+\mu} .
$$

Step 4. Conclusion. We summarize the different cases in Figure 1.

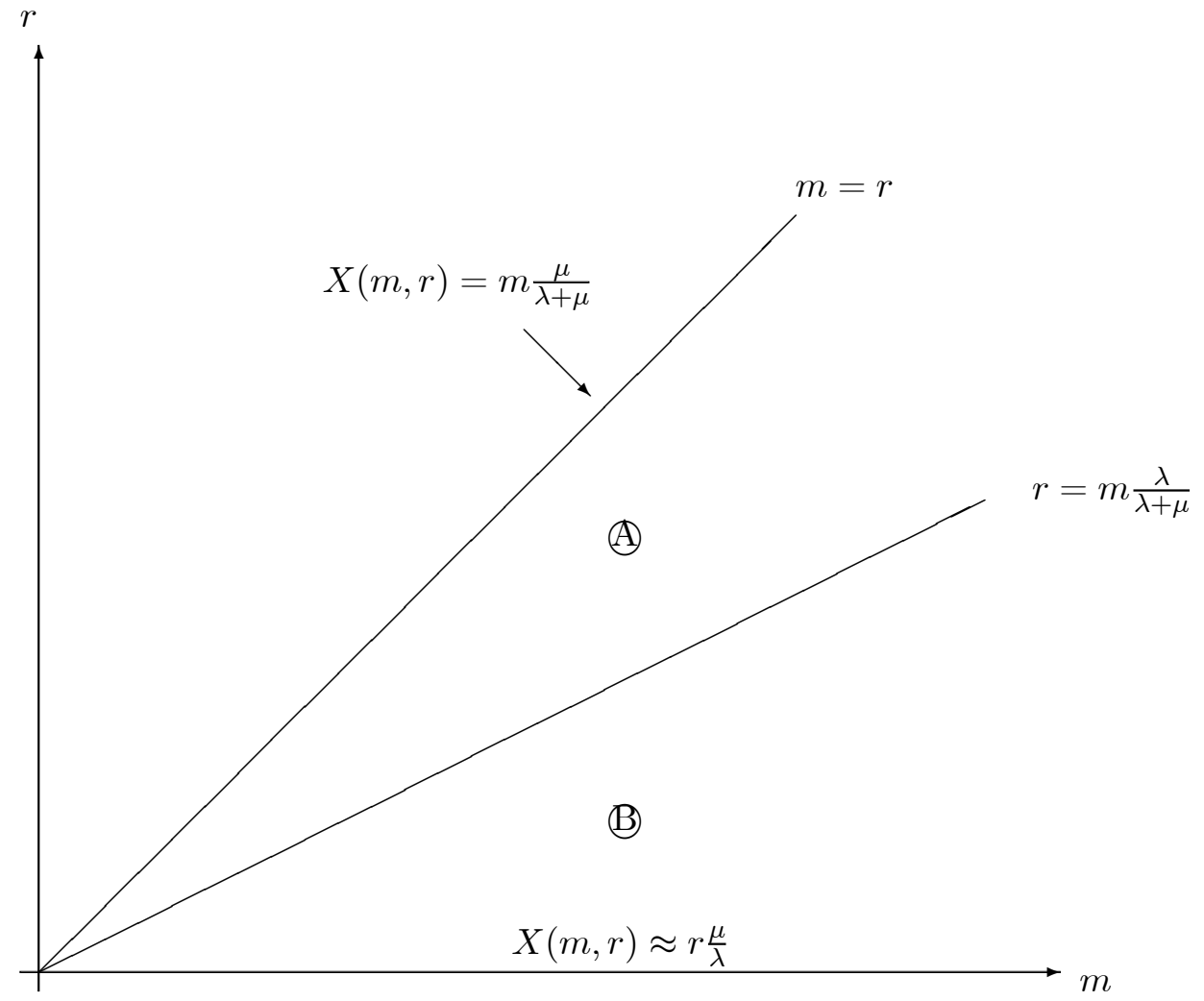

Figure 1: COMBINATIONS OF MACHINES AND REPAIRMEN 
Remember we only consider the cases where $r \leq m$. Zone (A) corresponds to $m \geq r \geq m \frac{\lambda}{\lambda+\mu}$, and zone (B) corresponds to $0 \leq r \leq m \frac{\lambda}{\lambda+\mu}$. Assume the approximations of the previous steps are correct.

Let $(m, r)$ be in zone (A), that is $r \geq m \frac{\lambda}{\lambda+\mu}$. We first have $X(m, r) \leq X(m, m)=m \frac{\mu}{\lambda+\mu}$. We also have $X(m, r) \geq X\left(m, m \frac{\lambda}{\lambda+\mu}\right)$, and the point $\left(m, m \frac{\lambda}{\lambda+\mu}\right)$ is on the line $r=m \frac{\lambda}{\lambda+\mu}$ so by step 3, $X\left(m, m \frac{\lambda}{\lambda+\mu}\right) \approx m \frac{\mu}{\mu+\lambda}$. Consequently, if $(m, r)$ belongs to zone $\AA$,

$$
X(m, r) \approx m \frac{\mu}{\mu+\lambda}=\varphi(m, r)
$$

Let $(m, r)$ be in zone (B), that is $r \leq m \frac{\lambda}{\lambda+\mu}$. We have $X(m, r) \leq \lim _{m^{\prime} \rightarrow \infty} X\left(m^{\prime}, r\right)$, and by step 2 the limit is $r \frac{\mu}{\lambda}$. Finally $X(m, r) \geq X\left(r \frac{\lambda+\mu}{\lambda}, r\right)$, and the point $\left(r \frac{\lambda+\mu}{\lambda}, r\right)$ is on the critical line so by step 3, $X\left(r \frac{\lambda+\mu}{\lambda}, r\right) \approx r \frac{\mu}{\lambda}$. Consequently, if $(m, r)$ belongs to zone $\left.\mathbb{B}\right)$,

$$
X(m, r) \approx r \frac{\mu}{\lambda}=\varphi(m, r)
$$

In all cases we have obtained:

$$
X(m, r) \approx \varphi(m, r) .
$$

\section{Remark}

The model does not take into account the possibility for repairmen to attend a machine before it fails. ${ }^{4}$ An alternative model could assume that, if at some period a repairman is not repairing a broken machine, then he takes care of a working machine, with the consequence that this machine has a lower probability of breaking down for the next period, a nil probability in what follows. With the same notations, the transitions would then read: If $m-n \geq r$, all repairmen are servicing broken machines, so: $\mathbb{P}(n-1 \mid n)=\lambda n$ and $\mathbb{P}(n+1 \mid n)=r \mu$. And if $m-n<r$, there are $m-r$ working machines not serviced, so $\mathbb{P}(n-1 \mid n)=\lambda(m-r)$ and all broken machines are being repaired, so $\mathbb{P}(n+1 \mid n)=(m-n) \mu$. The analog $X^{\prime}(m, r)$ of $X(m, r)$ (long-term expected number of machines working) would then naturally be higher.

For instance, in the case where $r=m$, the transitions would read: $\mathbb{P}(n-1 \mid n)=0$, and $\mathbb{P}(n+1 \mid n)=$ $(m-n) \mu$. The chain is not ergodic in this special case, and the number of machines working converges almost surely to $X^{\prime}(m, r)=m>X(m, r)=m \frac{\mu}{\lambda+\mu}$. In the case where $r=m-1$, we have for $n \geq 1$ : $\mathbb{P}(n-1 \mid n)=\lambda$ (exactly one working machine is not taken care of), and $\mathbb{P}(n+1 \mid n)=(m-n) \mu$. The equilibrium point is given by $(m-n) \mu=\lambda$, and one can show that $X^{\prime}(m, r) \simeq m-\frac{\lambda}{\mu}$. On the contrary, if $r$ is not too big then the variant would not bring significant differences: if $r=m \frac{\mu}{\lambda+\mu}$, we would obtain as in the original model $\frac{X(m, r)}{\varphi(m, r)} \underset{r \rightarrow \infty}{\longrightarrow} 1$. This is quite intuitive: since if $r$ is small only very few repairman will be serving working machines.

In the following section, we do not consider this alternative hypothesis.

\footnotetext{
${ }^{4}$ We are indebted to an anonymous referee of the journal for this remark.
} 


\section{Economic Consequences}

We first study the shape of the cost function for a fleet of $X$ machines in working mode. Secondly we study the consequences of a constraint for the operators that the avaibility of each machine should not be lower than a given threshold.

\subsection{Cost of a Fleet of Machines in Working Mode}

The convergence of $X(m, r)$ to

$$
\varphi(m, r)=\min \left\{r \frac{\mu}{\lambda}, m \frac{\mu}{\mu+\lambda}\right\}
$$

for large values of $m$ and $r$ has significant economic implications, especially for the electricity industry at a time when governments encourage the proliferation of decentralized production units represented here by the number $m$. In order to explain these implications simply, let us assume that (2) is an exact value instead of a limit. ${ }^{5}$ The relation (2) establishes a strict complementarity between inputs. It is represented by the L-shaped isoquants in Figure 2.

Under this type of technology, if we want to benefit from a large number of operational production units $X(m, r)$ or maximize the individal rate of availability $\alpha(m, r)$, we must deploy a dense network of repairmen $r$. The proliferation of dispersed units results not only in the creation of many jobs, which is a clear social advantage (especially at the concerned locations) but also in an increase in production costs. Indeed, the technology represented by (2) is a Hicks-Leontiev production function. ${ }^{6}$ It clearly shows that, regardless of unit $\operatorname{costs} c_{m}$ and $c_{r}$ of machines ${ }^{7}$ and repairers respectively, a single technical combination is efficient to get $X$ machines functioning. It is given by

$$
r \frac{\mu}{\lambda}=m \frac{\mu}{\mu+\lambda}=X
$$

In this efficient combination, we have $\frac{r}{m}=\frac{\lambda}{\mu+\lambda}<1$. Clearly we do not need as many repairmen as there are installed machines. With $r>m \frac{\lambda}{\mu+\lambda}$, some repairmen would not be used ${ }^{8}$, and with $r \frac{\mu+\lambda}{\lambda}<m$ some machines would remain down due to the lack of repairmen. To efficiently attain a number $X$ of operational machines, we need to install $m=\frac{\mu+\lambda}{\mu} X$ machines and have $r=\frac{\lambda}{\mu} X$ repairers. We can deduce that the cost to have $X$ machines in operating condition is

$$
C(X)=\left(\frac{\lambda}{\mu} c_{r}+\frac{\mu+\lambda}{\mu} c_{m}\right) X
$$

\footnotetext{
${ }^{5}$ As we have already seen, for $m$ and $r$ given the average production $X(m, r)$ is approximated very quickly because of the geometric convergence of the iterated Markov chain transition matrix. Furthermore, since $X(m, r) \leq \varphi(m, r)$, reasoning on $\varphi(m, r)$ to calculate the cost function, we underestimate the true cost of the average fleet $X(m, r)$.

${ }^{6}$ This function with strictly complementary inputs can be derived from a CES (Constant Elasticity of Substitution) function where the substitutability coefficient tends towards 0 .

${ }^{7}$ If $c_{r}$ stands for annual wage, the unit $\operatorname{cost} c_{m}$ must include the annuity for depreciation on top of operating costs.

${ }^{8}$ Note that if $r \geq m$, any down machine is serviced by a repairman and $r-m$ repairers are permanently inactive. However, $m>r>m \frac{\lambda}{\mu+\lambda}$ does not mean that $r-m \frac{\lambda}{\mu+\lambda}$ repairers are always inactive. On average there are too many repairmen, but they are useful in some unlikely instances where a very large number of machines are down.
} 


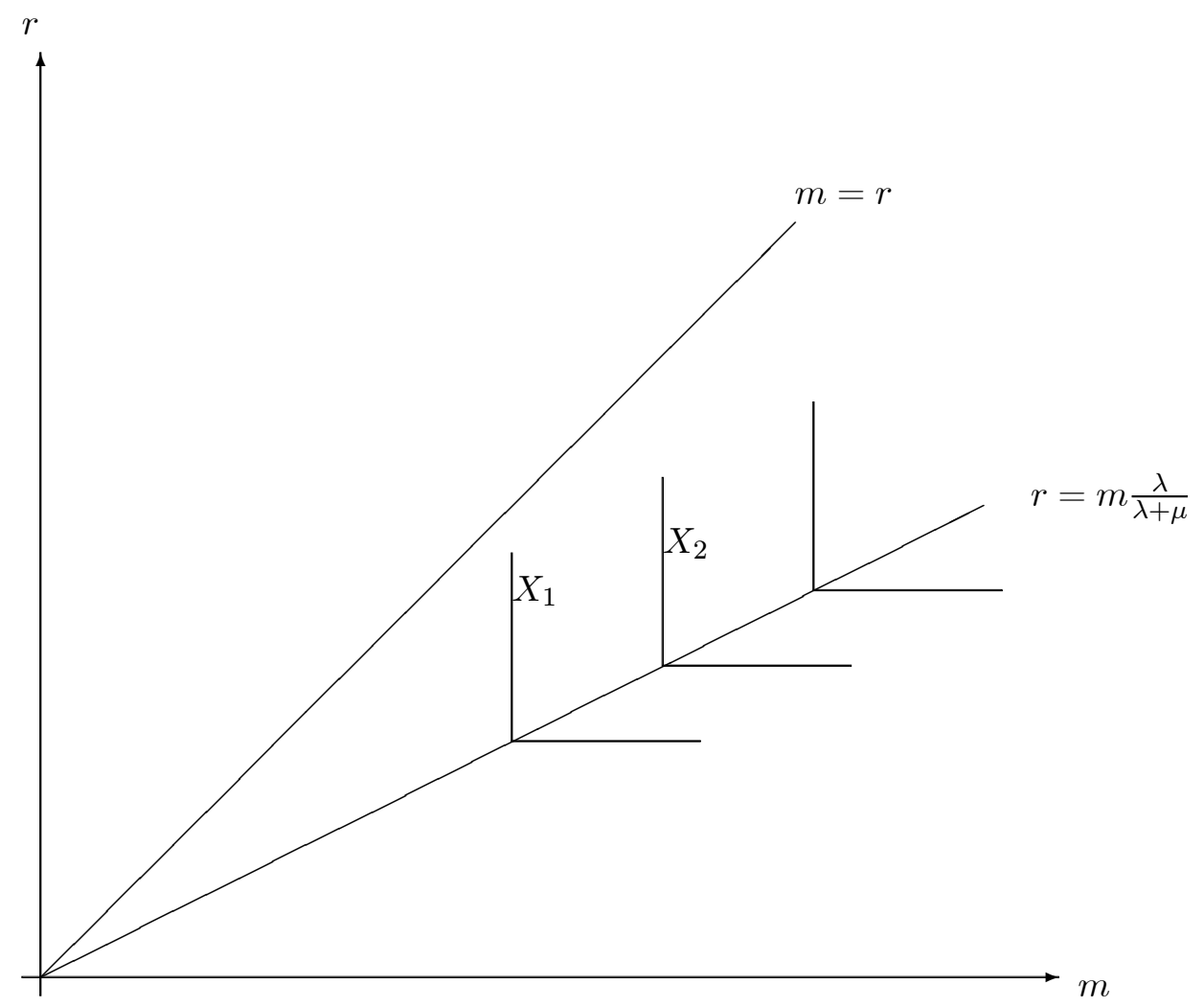

Figure 2: $L$-SHAPED ISOQUANTS $\left(X_{2}>X_{1}\right)$.

that is an average cost per working machine

$$
\frac{C(X)}{X}=c_{m}+\frac{\lambda}{\mu}\left(c_{r}+c_{m}\right)
$$

This formula shows explicitly that the purchase cost of a machine $c_{m}$ is only a part of the true cost of a machine in working condition. The additional cost $\frac{\lambda}{\mu}\left(c_{r}+c_{m}\right)$ is for transforming an "installed machine" into a "working machine" ${ }^{9}$. Reducing this cost necessitates an increase in R\&D to lower the ex ante probability of failure $\lambda$ and to improve the training of repairmen so that their efficiency $\mu$ increases. It also shows that if there is fierce competition on the machine market leading to a $\$ 1$ drop in the unit purchase price $c_{m}$, the price decrease is amplified for the buyers since the unit cost of a working machine will be decreased by $\$\left(1+\frac{\lambda}{\mu}\right)>\$ 1$. For example, during the past decade, economies of scale and learning-by-doing have fed the competition process in both the photovoltaic panels industry and the wind turbines industry. Because all price cuts were amplified at the buyer level, subsidized demand for PV panels and wind turbines increased more rapidly than governments

\footnotetext{
${ }^{9}$ For the machine to produce, we also need raw materials, manpower and so on. In the case of decentralized units to produce electricity from intermittent energy sources, wind and solar energy introduce an additional layer of randomness.
} 
expected, provoking what some have called an "existential threat" to Europe's electricity providers ${ }^{10}$.

\subsection{Availability Constraint}

Public authorities and standardization bodies often set quantitative targets in order to force manufacturers to provide minimum quality levels, primarily to maximize availability duration. ${ }^{11}$ In our model framework, it would consist in requiring that the availability rate of $m$ installed machines, $\alpha(m, r)$, not go below a given threshold $\alpha<1$. What is the number of repairmen necessary to keep each of these $m$ machines in working mode for at least a percentage $\alpha$ of the time?

We still assume that (2) is an exact value. Starting from Theorem (2.2), the availability constraint can be written

or

$$
\frac{\varphi(m, r)}{m} \geq \alpha
$$

$$
\min \left\{\frac{r}{m} \frac{\mu}{\lambda}, \frac{\mu}{\mu+\lambda}\right\} \geq \alpha
$$

We first observe that, for a given technology characterized by $\mu$ and $\lambda$, the percentage $\frac{\mu}{\mu+\lambda}$ is the maximal value that can be fixed for the threshold constraint. Fixing a value $\alpha>\frac{\mu}{\mu+\lambda}$ is a non feasible requirement. In fact, given Theorem (2.2) and the result obtained at step 1 of the heuristic proof, we can write that $\alpha(m, r)=\frac{X(m, r)}{m} \leq \frac{X(m, m)}{m}=\frac{\mu}{\mu+\lambda}$ for any value of $m$ and $r$. It follows that $\frac{\mu}{\mu+\lambda}$ is the exact value to which the availabilty rate converges.

This affords a better understanding of what occurs when the threshold $\alpha$ is not too large. From (5) the number of installed machines and the number of repairers must be related through the inequality $\frac{r}{m} \frac{\mu}{\lambda} \geq \alpha$. Actually, this constraint is not binding for the efficient combination $(m, r)$ chosen by the installers since the constraint's frontier is given by

$$
r=\frac{\lambda \alpha}{\mu} m
$$

Now, the installer is always better off choosing the combination $(m, r)$ that minimizes costs. By (3), as shown in Figure 1, we know that this combination is determined by

$$
r=\frac{\lambda}{\mu+\lambda} m
$$

Since $\alpha<\frac{\mu}{\mu+\lambda}$, one can easily verify that the efficient combination always satifies the availability constraint. Geometrically, in Figure 1 the line defined by (6) is below (if $\alpha<\frac{\mu}{\mu+\lambda}$ ) or merged with (if $\alpha=\frac{\mu}{\mu+\lambda}$ ) the efficient ray defined by (7). In other words, if they are in charge of maintenance, installers 'naturally' choose to hire at least as many repairmen as a feasible quality regulation might require them to.

In summary, in the current framework, fixing a minimum value for the availability rate is inadequate regulation in so far as at best it is a redundant constraint (when $\alpha$ is small) and at worst the target is out of reach (when $\alpha$ is large).

\footnotetext{
${ }^{10}$ The Economist, October 12th 2013, "European utilities. How to lose half a trillion euros"

${ }^{11}$ See for example Crampes and Hollander [1995].
} 
Nevertheless, Minimum Quality Standard can be adequate under other circumstances. For example, it can trigger investment in quality improvement: a large target $\alpha$ can be justified in the case where the installer is able to modify $\lambda$ and $\mu$ by investing to improve the performance of machines and repairers. If they face a penalty everytime $\alpha-\frac{\mu}{\mu+\lambda}>0$ is observed, installers will seek to increase $\mu$ by training programmes and to lower $\lambda$, both ex ante by R\&D expenditures and ex post by regular maintenance visits. Also note that the necessity to improve the ratio $\frac{\mu}{\mu+\lambda}$ is a natural result in a competition framework if entry is easy. But if the quality levels resulting from competition are viewed as too low on safety or reliability grounds, a Minimum Quality Standard helps in selecting the entrants.

\section{Conclusions}

Starting from a probabilistic model set up by ARROw et al. [1972], we have determined the cost of a machine in working mode as a combination of the installed machine cost, the cost of reparations after failure, the probability of failure and the probability of a successful repair. The linear cost function is derived from the basic result of the model, which is that machines and repairmen must be viewed as complements to reach a given level of production when that level is high. The analysis is based on strong simplifying assumptions, especially (i) mending operations are independent over time, and (ii) simultaneous changes are negligible, which may be more and more difficult to hold when $m$ and $r$ grow. Nevertheless, the basic result would most likely be reinforced if we relax these hypotheses, particularly if we introduce some positive correlation between the probabilities of individual failure (e.g. a storm damaging all the turbines in a windfarm ${ }^{12}$ ).

In all industries, the extended cost of an operational machine is essential information for both private investors and public decision-makers. The precise information on actual maintenance costs is missing for most small-scale plants. In the framework of environmental and energy policy for instance, wind turbines (with capacity between a handful of $\mathrm{kW}$ and $5 \mathrm{MW}$ for the largest) can challenge thermal, hydro and nuclear plants (between 1 and $1.5 \mathrm{GW}$ for the latter) only by investing in a large number of facilities, especially because the wind rarely blows more than $50 \%$ of the time. Some mechanical parts of turbines must regularly be checked and replaced. The Arrow's model we have revisited shows that the number of repairmen required for maintaining the turbines increase in proportion with the number of installed turbines (for a given technology). This result should facilitate the computation of actual operating costs of large fleets of small plants, in particular scattered plants producing energy from renewable sources.

Acknowledgements: The authors thank two anonymous referees of the Annals of Economics and Statistics for their constructive remarks.

Claude Crampes (corresponding author), Toulouse School of Economics (GREMAQ, Université Toulouse 1 Capitole), claude.crampes@tse-fr.eu, UT1-Capitole, 21 allée de Brienne, 31000 Toulouse

Jérôme Renault, Toulouse School of Economics (GREMAQ, Université Toulouse 1 Capitole), jerome.renault@tse-fr.eu, UT1-Capitole, 21 allée de Brienne, 31000 Toulouse

\footnotetext{
${ }^{12}$ For example, see www.kumeyaay.com/all-news/439-wind-farm-a-damaging-blow.html and www.eastcountymagazine.org/node/2734.
} 


\section{Appendix}

In this appendix we give a formal proof of Theorem 2.2. We start with the proof of Part (3), and we also obtain Part (2) at the end of step $2^{*}$ of the proof. Finally we prove Part (1).

\subsection{Proof of $\lim _{r \rightarrow \infty} \frac{X(m, r)}{\varphi(m, r)}=1$.}

We essentially follow the steps of the heuristic proof.

Step $1^{*}$. The case $r=m$.

Here, the transition matrix reads:

$$
\begin{aligned}
& \begin{array}{lllllllllll}
0 & 1 & 2 & \ldots & n & \ldots & \ldots & \ldots & m
\end{array}
\end{aligned}
$$

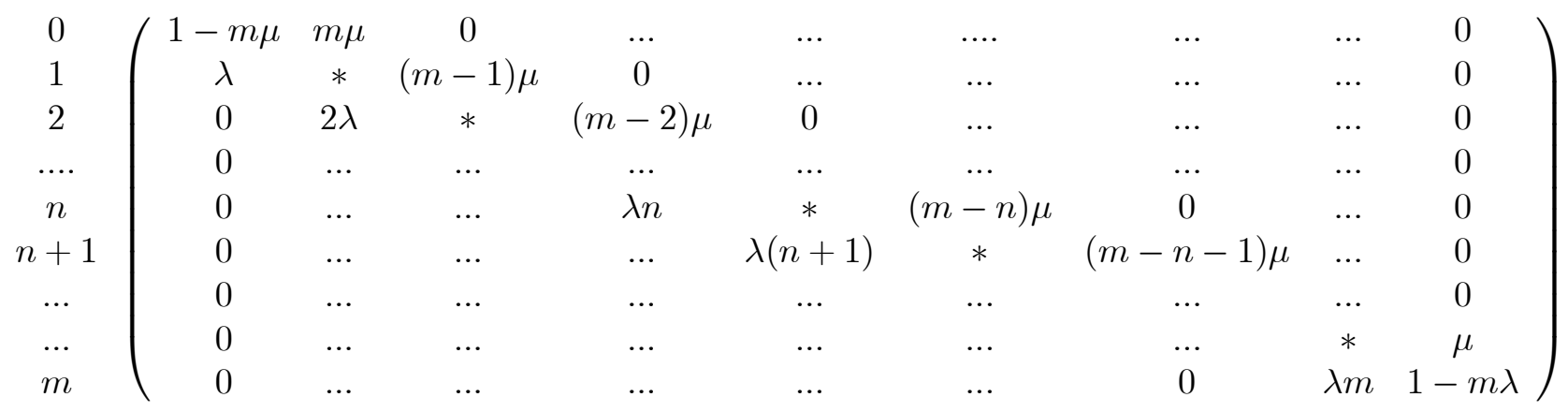

Solving for $p H=p$, we obtain that for all $n$ in $\{1, \ldots, m\}$ :

$$
(m+1-n) \mu p_{n-1}+(1-\lambda n-(m-n) \mu) p_{n}+\lambda(n+1) p_{n+1}=p_{n} .
$$

So $(m+1-n) \mu p_{n-1}+\lambda(n+1) p_{n+1}=(\lambda n+(m-n) \mu) p_{n}$, and by an easy induction we obtain that for all $n \geq 1$,

$$
n \lambda p_{n}=p_{n-1}(m-n+1) \mu=m p_{n-1} \mu-(n-1) p_{n-1} \mu .
$$

We sum the previous equality for $n=1$ to $n=m$, and obtain:

$$
\lambda X(m, r)=m \mu\left(1-p_{m}\right)-\mu\left(X(m, r)-m p_{m}\right) .
$$

We obtain that in this case where $r=m$,

$$
X(m, r)=m \frac{\mu}{\lambda+\mu} .
$$

And it implies that for any possible values of $m$ and $r: X(m, r) \leq X(m, m)=m \frac{\mu}{\lambda+\mu}$. 
Step $2^{*}$. The upper bound $\frac{r \mu}{\lambda}$

If $r$ is small, the $r$ repairmen will be working most of the time. It is therefore natural to consider the modified transition matrix $H^{\prime}$ :

$$
\left.\begin{array}{cccccccccc}
\multicolumn{1}{c}{0} & 1 & 2 & \ldots & n & \ldots & \ldots & \ldots & m \\
0 & & & & & & & & & \\
1 & 1-r \mu & r \mu & 0 & \ldots & \ldots & \ldots & \ldots & \ldots & 0 \\
2 & \lambda & * & r \mu & 0 & \ldots & \ldots & \ldots & \ldots & 0 \\
\ldots & 0 & 2 \lambda & * & r \mu & 0 & \ldots & \ldots & \ldots & 0 \\
n & 0 & \ldots & \ldots & \ldots & \ldots & \ldots & \ldots & \ldots & 0 \\
n+1 & 0 & \ldots & \ldots & \lambda n & * & r \mu & 0 & \ldots & 0 \\
\ldots & 0 & \ldots & \ldots & \ldots & \lambda(n+1) & * & r \mu & \ldots & 0 \\
\ldots & 0 & \ldots & \ldots & \ldots & \ldots & \ldots & \ldots & \ldots & 0 \\
m & 0 & \ldots & \ldots & \ldots & \ldots & \ldots & \ldots & * & r \mu \\
& 0 & \ldots & \ldots & \ldots & \ldots & \ldots & 0 & \lambda m & 1-m \lambda
\end{array}\right)
$$

We denote the unique invariant measure for $H^{\prime}$ by $p^{\prime}$ and by $X^{\prime}(m, r)=\sum_{n=0}^{m} n p_{n}^{\prime}$. Note that $H^{\prime}$ represents a better situation for production than $H$ since we have increased several probabilities of going upwards, so $X^{\prime}(m, r) \geq X(m, r)$.

Solving for $p^{\prime} H^{\prime}=p^{\prime}$, we obtain that for all $n$ in $\{1, \ldots, m\}$ :

$$
r \mu p_{n-1}^{\prime}+(1-\lambda n-r \mu) p_{n}^{\prime}+\lambda(n+1) p_{n+1}^{\prime}=p_{n}^{\prime} .
$$

So $r \mu p_{n-1}^{\prime}+\lambda(n+1) p_{n+1}^{\prime}=(\lambda n+r \mu) p_{n}^{\prime}$, and by an easy induction we obtain that for all $n \geq 1$,

$$
n \lambda p_{n}^{\prime}=p_{n-1}^{\prime} r \mu .
$$

Taking the sum from $n=1$ to $n=m$, we get $\lambda X^{\prime}(m, r)=r \mu\left(1-p_{m}^{\prime}\right)$, so:

$$
X(m, r) \leq X^{\prime}(m, r)=r \frac{\mu}{\lambda}\left(1-p_{m}\right) \leq r \frac{\mu}{\lambda} .
$$

Note that the above expression is true for all $m$ and $r$. Combined with the last line of step $1^{*}$, we already obtain point (2) of theorem 2.2 , that $X(m, r) \leq \varphi(m, r)$ for all $0 \leq r \leq m$.

Step $3^{*}$. General case.

We solve for $p H=p$. We obtain that for all $n$ in $\{1, \ldots, m-r\}$ :

$$
r \mu p_{n-1}+(1-\lambda n-r \mu) p_{n}+\lambda(n+1) p_{n+1}=p_{n} .
$$

So $r \mu p_{n-1}+\lambda(n+1) p_{n+1}=(\lambda n+r \mu) p_{n}$, and by an easy induction we obtain:

$$
\forall n \in\{1, \ldots, m-r\}, n \lambda p_{n}=p_{n-1} r \mu .
$$


Taking the sum from $n=1$ to $n=m-r$, we get $\lambda \sum_{n=0}^{m-r} n p_{n}=r \mu\left(1-\sum_{n=m-r}^{m} p_{m}\right)$, so:

$$
\lambda X(m, r)=r \mu-r \mu \sum_{n=m-r}^{m} p_{n}+\lambda \sum_{n=m-r+1}^{m} n p_{n} .
$$

Thus

$$
X(m, r)=\frac{r \mu}{\lambda}-p_{m-r} \frac{r \mu}{\lambda}+\sum_{n=m-r+1}^{m}\left(n-\frac{r \mu}{\lambda}\right) p_{n} .
$$

Taking the product from $n=1$ to $n=m-r$ of equations (8), we obtain:

$$
p_{m-r}=p_{0} \frac{\left(\frac{r \mu}{\lambda}\right)^{m-r}}{(m-r) !} .
$$

Thus

$$
X(m, r)=\frac{r \mu}{\lambda}-p_{0} \frac{\left(\frac{r \mu}{\lambda}\right)^{m+1-r}}{(m-r) !}+\sum_{n=m-r+1}^{m}\left(n-\frac{r \mu}{\lambda}\right) p_{n} .
$$

Step $4^{*}$. The case of small $r$.

If $(m, r)$ is in zone (B), we have $n-\frac{r \mu}{\lambda} \geq 0$ for all $n \geq m-r+1$, so in this case combining steps $2^{*}$ and $3^{*}$ we get:

$$
\frac{r \mu}{\lambda} \geq X(m, r) \geq \frac{r \mu}{\lambda}-\frac{\left(\frac{r \mu}{\lambda}\right)^{m+1-r}}{(m-r) !}
$$

This implies that $\lim _{m \rightarrow \infty} X(m, r)=\frac{r \mu}{\lambda}$.

Step $5^{*}$. The case of $r=m \frac{\lambda}{\lambda+\mu}$.

In this case $m-r=m \frac{\mu}{\mu+\lambda}=r \frac{\mu}{\lambda}$. We are on the critical line, so formally $(m, r)$ belongs to zone (B) and we can apply the previous inequalities: $\frac{r \mu}{\lambda} \geq X(m, r) \geq \frac{r \mu}{\lambda}-\frac{\left(\frac{r \mu}{\lambda}\right)^{m+1-r}}{(m-r) !}$. However $\frac{(m-r)^{m-r}}{(m-r) !}$ converges ${ }^{13}$ to $+\infty$ when $m-r$ goes to $+\infty$, so we have to be more precise. Denote by $\gamma \in(0,1)$ the following ratio:

$$
\gamma={ }_{\text {def }} \frac{\mu}{\lambda+\mu} .
$$

We have $m-r=m \gamma$, and we know by equation (9) that $m \gamma \geq X(m, r) \geq m \gamma-p_{m-r} m \gamma$. To show that $\frac{X(m, r)}{m \gamma}$ is close to 1 , we now show that $p_{m-r}$ is small.

\footnotetext{
${ }^{13}$ Recall Stirling's formula:
}

$$
n ! \sim n^{n} e^{-n} \sqrt{2 \pi n} .
$$


For all $n \leq m-r$, multiplying equations (8) from 1 to $n$, we get:

$$
p_{n}=p_{0} \frac{(m \gamma)^{n}}{n !}
$$

This implies that for all $n \leq m-r$ :

$$
p_{n}=p_{m-r} \frac{(m \gamma) !}{(m \gamma)^{m \gamma-n} n !} .
$$

So

$$
\sum_{n=0}^{m-r} p_{n}=\frac{p_{m-r}(m \gamma) !}{e^{-m \gamma}(m \gamma)^{m \gamma}} \sum_{n=0}^{m \gamma} \frac{(m \gamma)^{n}}{n !} e^{-m \gamma} .
$$

Consider the sum $\sum_{n=0}^{m \gamma} \frac{(m \gamma)^{n}}{n !} e^{-m \gamma}$. If $Z$ is a Poisson random variable with parameter $(m \gamma)$, this is nothing but the probability $\mathbb{P}(Z \leq m \gamma)$. Knowing that the median of a Poisson distribution with parameter $\theta$ is at most $\theta+1 / 3$, we infer that $\mathbb{P}(Z \leq m \gamma) \geq 1 / 2$. Since $\sum_{n=0}^{m-r} p_{n} \leq 1$, we get:

$$
p_{m-r} \leq 2 \frac{e^{m \gamma}(m \gamma)^{m \gamma}}{(m \gamma) !} \sim \frac{2}{\sqrt{2 \pi m \gamma}}
$$

Consequently, if $m$ is large enough, we have $p_{m-r} \leq \frac{3}{\sqrt{2 \pi m \gamma}}$, which implies by equation (9):

$$
X(m, r) \geq m \gamma\left(1-\frac{3}{\sqrt{2 \pi m \gamma}}\right) .
$$

\section{Step $6^{*}$. Conclusion}

Let $(m, r)$ be in zone (A). We first have $X(m, r) \leq X(m, m)=m \frac{\mu}{\lambda+\mu}$ by step $1^{*}$. We also have $X(m, r) \geq X\left(m, m \frac{\lambda}{\lambda+\mu}\right)$, and the point $\left(m, m \frac{\lambda}{\lambda+\mu}\right)$ is on the critical line $r=m \frac{\lambda}{\lambda+\mu}$ so by step $5^{*}$, if $m$ is large enough then $X\left(m, m \frac{\lambda}{\lambda+\mu}\right) \geq m \gamma\left(1-\frac{3}{\sqrt{2 \pi m \gamma}}\right)$, where $\gamma=\frac{\mu}{\lambda+\mu}$. We have $m \gamma=m \frac{\mu}{\lambda+\mu}=\varphi(m, r)$ here so we obtain: if $(m, r)$ belongs to zone $\AA$ ) and $m$ is large enough,

$$
1 \geq \frac{X(m, r)}{\varphi(m, r)} \geq 1-\frac{3}{\sqrt{2 \pi \varphi(m, r)}}=1-\frac{3 \sqrt{\lambda+\mu}}{\sqrt{2 \pi m \mu}} .
$$

Now let $(m, r)$ be in zone (B). $\varphi(m, r)=\frac{r \mu}{\lambda}$, and we first have $X(m, r) \leq \lim _{m^{\prime} \rightarrow \infty} X\left(m^{\prime}, r\right)=\frac{r \mu}{\lambda}$. We also have $X(m, r) \geq X\left(m^{\prime}, r\right)$, where $m^{\prime}=r \frac{\lambda+\mu}{\lambda} \leq m$. By step $5^{*}$, we obtain that if $r$ is large enough, $X\left(m^{\prime}, r\right) \geq m^{\prime} \gamma\left(1-\frac{3}{\sqrt{2 \pi m^{\prime} \gamma}}\right)$, with $m^{\prime} \gamma=r \frac{\mu}{\lambda}$. So we obtain: if $(m, r)$ belongs to zone (B) and $r$ is large enough,

$$
1 \geq \frac{X(m, r)}{\varphi(m, r)} \geq 1-\frac{3}{\sqrt{2 \pi \varphi(m, r)}}=1-\frac{3 \sqrt{\lambda}}{\sqrt{2 \pi r \mu}}
$$

We obtain :

$$
\lim _{r \rightarrow \infty} \frac{X(m, r)}{\varphi(m, r)}=1
$$




\subsection{Proof of $\alpha(m, r)=\frac{X(m, r)}{m}$.}

Let $K=\{0,1\}^{M}$ denote the set of possible positions of our system. Given an element $y=\left(y_{1}, \ldots, y_{m}\right)$ in $K$, for each $i$ in $\{1, \ldots, m\}$ the variable $y_{i}=1$ is interpreted as "machine $i$ is working" whereas $y_{i}=0$ is interpreted as "machine $i$ is in failure mode". We consider here the process $\left(Y^{t}\right)_{t \geq 0}$ where for each machine $i$ and time $t \geq 0, Y_{i}^{t}$ is the random variable in $\{0,1\}$ indicating whether machine $i$ is working or in failure mode at time $t$. $\left(Y^{t}\right)_{t \geq 0}$ is a Markov chain taking values in $K$.

Assuming that $r \geq 1$ (see footnote 3 ), given $y$ and $y^{\prime}$ in $K$ there is a positive probability to reach $y^{\prime}$ from $y$ in exactly $m$ stages. Hence the Markov chain $\left(Y^{t}\right)_{t \geq 0}$ is ergodic and there is a unique probability $\Pi$ on $K$ which is stationary for the Markov chain. By applying the ergodic theorem ${ }^{14}$, for any initial position, for each $y$ in $K$, with probability we have that:

$$
\frac{1}{T} \sum_{t=1}^{T} \mathbf{1}_{Y^{t}=y} \underset{T \rightarrow \infty}{\longrightarrow} \Pi(y) .
$$

Note that there is a clear link between $\Pi$ and the invariant measure $p^{*}=\left(p_{0}^{*}, \ldots, p_{m}^{*}\right)$ introduced in Section 2, which gives the long-term frequency of the number of machines working. For each $y=\left(y_{1}, \ldots, y_{m}\right)$ in $K$, by the symmetry across machines $\Pi(y)$ only depends on the number of machines working, that is, on $n(y)={ }_{\text {def }} \sum_{i=1}^{m} y_{i}$. Fixing the number $n$ of working machines, there are exactly $\left(\begin{array}{l}m \\ n\end{array}\right)=\frac{m !}{n !(m-n) !}$ configurations $y$ such that $n(y)=n$. And we have:

$$
\Pi(y)=\frac{p_{n(y)}^{*}}{\left(\begin{array}{c}
m \\
n(y)
\end{array}\right)} .
$$

Assume now we fix a machine $i$, and let $K_{i}$ be the set of $y$ in $K$ such that $y_{i}=1$. Using equation (10), we obtain that almost surely the frequency of machine $i$ working converges:

$$
\frac{1}{T} \sum_{t=1}^{T} \mathbf{1}_{Y_{i}^{t}=1}=\frac{1}{T} \sum_{t=1}^{T} \sum_{y \in K_{i}} \mathbf{1}_{Y^{t}=y} \underset{T \rightarrow \infty}{\longrightarrow} \sum_{y \in K_{i}} \Pi(y) .
$$

The probability under $\Pi$ that machine $i$ is working is $A_{i}=\sum_{y \in K_{i}} \Pi(y)$, which by symmetry does not depend on $i$. When computing $\sum_{i=1}^{m} A_{i}$, for each $y$ in $K$ we add $\Pi(y)$ exactly $n(y)$ times, so we obtain:

$$
\sum_{i=1}^{m} A_{i}=\sum_{y \in K} n(y) \Pi(y),
$$

Using the fact that $A_{i}$ does not depend on $i$, we get:

$$
m A_{i}=\sum_{y \in K} n(y) \Pi(y)=\mathbb{E}_{\Pi}(n)=X(m, r) .
$$

\footnotetext{
14 "The ergodic theorem is a version of the strong law of large numbers general enough to apply to any system governed by probability laws that are invariant in time", Billingsley (1995) p. 311 and Theorem 24.1on p. 314.
} 
By equations (11) and (12), we conclude that with probability 1:

$$
\frac{1}{T} \sum_{t=1}^{T} \mathbf{1}_{Y_{i}^{t}=1} \underset{T \rightarrow \infty}{\longrightarrow} \frac{X(m, r)}{m} .
$$

Each machine will produce in the long run with frequency $\frac{X(m, r)}{m}$, so $\alpha(m, r)=\frac{X(m, r)}{m}$ and condition (2) of Theorem 2.2 is proved.

Remark 6.1. Equation (13) tells us that the convergence of the frequency with which machine $i$ is working will happen almost surely. Using concentration inequalities for Markov chains, e.g. the Hoeffding inequality (see Glynn and Ormoneit, 2002) would provide tractable upper bounds on the probability that $\frac{1}{T} \sum_{t=1}^{T} \mathbf{1}_{Y_{i}^{t}=1}$ is far from $\frac{X(m, r)}{m}$ for a given $T$.

\section{References}

[1] Arrow K.J., D. Levhari and E. Sheshinski (1972) "A Production Function for the Repairman Problem", The Review of Economic Studies, Vol. 39, No. 3 (July), 241-249

[2] Billingsley P. (1995) "Probability and Measure", Wiley Series in Probability and Mathematical Statistics

[3] Crampes C. and Hollander A (1995) "Duopoly and Quality Standards", European Economic Review 39: 71-82

[4] Feller, W. (1957), "An Introduction to Probability and Its Applications", 2nd ed., vol. 1. New York: Wiley.

[5] Gowrisankaran G., S.S. Reynolds and M. Samano, (2013) "Intermittency and the Value of Renewable Energy", University of Arizona, March 4

[6] Glynn P. and D. Ormoneit (2002), "Hoeffding's Inequality for Uniformly Ergodic Markov Chains", Statistics and Probability Letters 56, pp.143-146.

[7] Hameed Z., Y.S. Hong, Y.M. Cho, S.H. Ahn and C.K. Song (2009), "Condition Monitoring and Fault Detection of Wind Turbines and Related Algorithms: A Review", Renewable and Sustainable Energy Reviews 13 (2009) 1-39

[8] Levhari D. and E. Sheshinski (1970), "A Micro-Economic Production Function", Econometrica, May, vol. 38, $\mathrm{n}^{\circ} 3,559-573$.

[9] Syrquin M. (1972), "Returns to Scale and Substitutability in the Repairmen Problem", Econometrica, Vol. 40, No. 5, September, pp. 937-941 